\title{
The Idea for Colleges to Practice New Model of "Cloud Storage" for Archives
}

\author{
Ju WANG ${ }^{1, a}$, Chenggang WANG ${ }^{2, b}$, Xiaomeng LIU $3, a$ \\ ${ }^{1,3}$ Shandong Medical College, Jinan, Shandong, 250002, China \\ ${ }^{2}$ SIEMENS (China) Co., Ltd, Jinan, Shandong Province 250022, China \\ axiaoxianxue@sohu.com, ${ }^{\mathrm{b}}$ chenggang.wang@siemens.com
}

Keywords: college archives, cloud storage, the new model

\begin{abstract}
Compared to traditional file storage, "Cloud Storage" has the advantages of low cost, high efficiency and large storage volume etc, drawing more and more attention from public, but at the same time, it also has certain problem and risk. This paper describes in details the advantages and characteristics of "cloud storage" in college archives storage, moreover, regarding the potential problems and risk, put forward the concrete solutions.
\end{abstract}

\section{Introduction}

Archives witness the course of college reform and development, they are historical record and original information for colleges to manage education and teaching activities. Archives is not only the important guarantee for the college strategic decisions, but also the indispensable resources and necessary means for daily management.

However, along with the increasing of the college scale, the complexity and diversification of educational features, archives need more and more time and space to store and manage. How to provide a large storage space for archives information, to provide users with a convenient access platform. With the development of information technology, cloud storage technology has emerged outstandingly. In brief, "cloud storage" is a newly developing solution to put the storage resources in cloud for people to access. The users can easily access the data at any time, any place, through any device capable of networking connection to the cloud. At present, many overseas large listed companies such as Microsoft, Amazon and Google have introduced corresponding cloud storage platform service. If cloud storage technology can be applied to the field of archives management in colleges and universities, it will be a historic revolution for the archives cause.

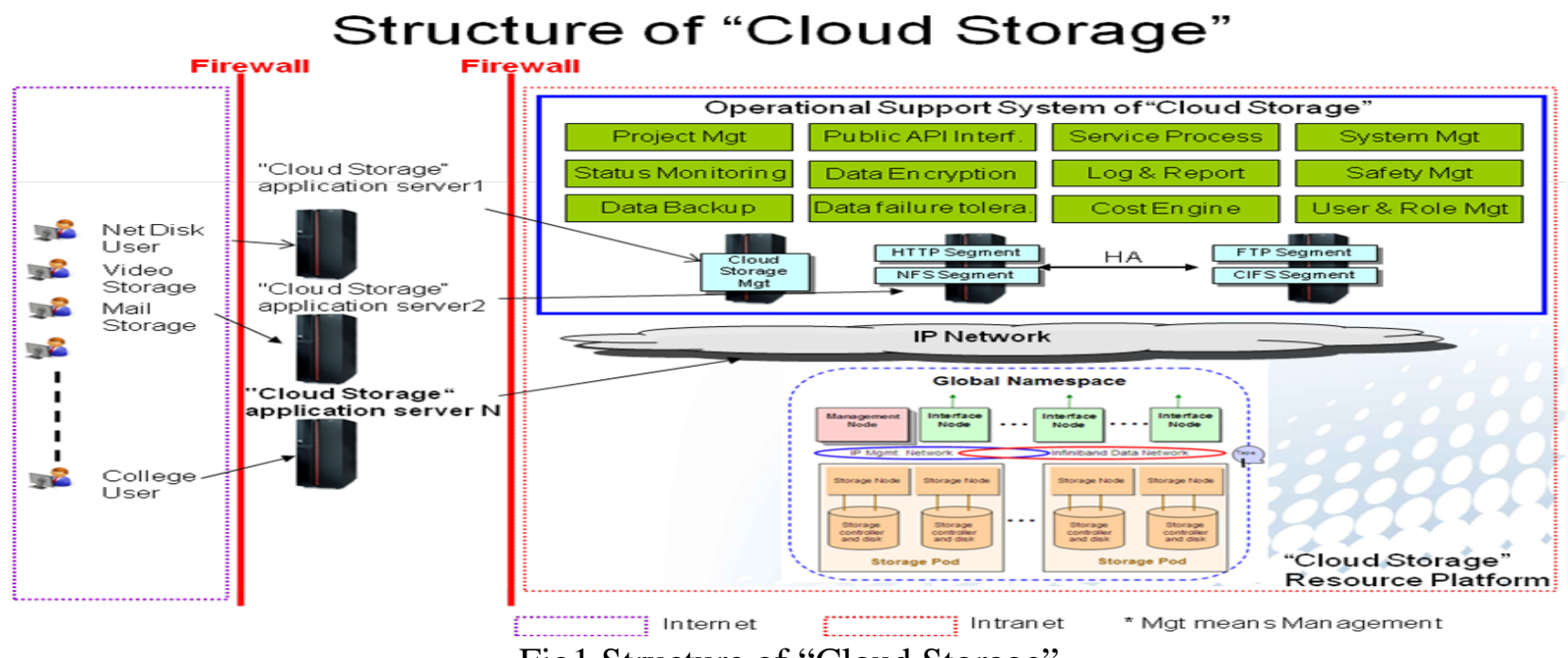

Fig1.Structure of "Cloud Storage”

The advantages of "Cloud storage" compared with traditional archives storage method in colleges 


\section{Reduce the cost of the archives management}

The traditional archive management mode needs a lot of financial support for the storage system, including hardware, software, updates, maintenance, upgrading etc, and needs the computer professionals to carry out the daily maintenance of servers and storage systems, also need give training to part-time archivists for their computer skill, that undoubtedly will be a huge overhead. But if the "cloud storage" technology will be used, the colleges can apply for "cloud storage" size according to the requirement of their actual development, it only needs very low initial investment, however, no needs to manage and maintain the storage system , also don't need to be equipped with computer professionals, the archivists can take more time to do their professional work instead of handling computer, which will reduce a lot of the cost of archives management.

\section{Improve the utilization efficiency of archives resources}

"Cloud storage" provides an open platform, not limited by time and place, it could realize the sufficient achieve resource share and access anytime and anywhere, solved the previous difficulty for some users unable to real-time access to archives due to the factors such as location or time etc, greatly improve the utilization efficiency of archives resources, the archives resources can truly be used anytime and anywhere.

\section{Expanded the storage space for archives resource}

Using "cloud storage" scheme, the digital file can be directly uploaded to the internet, so almost unnecessary to use the CD and other hardware resources, only a few computer terminals would be enough, all data are stored in the "cloud", "cloud" is composed of tens of thousands of units or more server cluster, and it has unlimited space and mass storage capability, and has the function of expansion, to meet the growing storage requirements of the university archives at present stage, greatly expanded the storage space of file resource.

\section{To strengthen safety of file storage}

With cloud technology, the collapse of a computer does not affect the data storage, because the cloud will automatically backup the storage data, and the central data storage is advantageous for the data center staff to unify the management of data,and real-time monitor, the user can get the maximum guarantee for data security. By the "Cloud storage", the archives resources will not be stored on a physical machine, but in the virtual "cloud" end, even if one or more machines into offline or malfunctioned, "cloud" can also continue to run normally, most of the data is still in, won't cause serious damage, could be completely and quickly restored on a new server, which will not affect the use of the whole, which greatly enhance the safety and reliability of file storage.

\section{The problems and risks of "Cloud storage" in archives management of colleges}

\section{File managers maybe lose the control over the files}

When the file information is handed over to "cloud storage" suppliers (hereinafter referred to as the "cloud") for processing and management, "cloud" has the right to access and control data, instead, the file managers will only have access right but lose control to the files, less likely to know which device are files to be stored, in which place, which piece of hard disk, and there is no way to enforce the protection of archives in person. If the "cloud" has not consider sufficiently the control rules of file and archive management, archives management will be out of control.

\section{Safety risk such as disclosure of archives information}

Archives secrecy determines the storage need a safe environment, the existing method of file storage has certain advantages in this aspect, but "cloud storage" technology is the product based on the Internet, the Internet's openness decides the threat of potential high-tech means such as hackers, network virus, or even from the internal management, under the condition of the current technical level, no one "cloud" has declared that it can provide an absolutely safe platform completely resisting various attacks. In addition, if the figures or documents are released publicly, which could not be opened or temporary could not be opened publicly, it could also lead to the leak of archives information, will cause serious consequence.

\section{Lack of reliable domestic cloud storage suppliers}


At present, most of successful "cloud" suppliers are foreign brands, such as Google and Microsoft cloud, although the domestic IT companies are struggling to pursue, but from the technical level and development scale, the existing gap is very big, most of them are carrying on the secondary development of foreign technology. When the college choose the "cloud", the "cloud" has control of file data, which will lead to the possible data theft behavior, or the behavior to dig useful and critical information through data mining techniques. So, to some extent, we hope to find a safe and reliable domestic "cloud" with independent R\&D qualification, for protection to the state secrets or unit secrets to the maximum extent.

\section{Lack of unified standard for cloud storage technology}

If the digital files need to be efficiently stored for a long term, a set of unified storage standard would be required. But, so far, around the world, there is still no recognized standard for long-term preservation of digital archive information, this may lead to some problems during the uploading process of digital archives to the "cloud end" , such as data loss, disorders etc , which would directly affect the integrity and systematicness of digital archives.

\section{"Cloud Archives" - The idea to implement the new mode of archive "cloud storage" in colleges}

\section{Actively participate in the design of "Cloud Archives", taking more initiative}

The colleges should establish an archives management system taking the archives management department as the leader, supported by other departments with individual responsibility, to avoid excessive reliance on "cloud" suppliers. In the resources construction and the core content design of management system, the colleges should take the lead as owner, establish safety standards and confidentiality agreement by themselves, all the archivists, including full-time and part-time, shall actively participate in the management system design of "cloud archives", learning the related principle and technologies of "cloud", then developing a reasonable scheme of archives management. In the procurement and system maintenance contracts with the "cloud" supplier, or even during daily cooperation, the strict confidentiality agreement must be established, and accordingly the tight security mechanism should be set up for mitigating risk to a minimum. The archives in the cloud platform on campus should be carefully made up and classified to determine which data can be deposited as "cloud archives", and what data should still be kept in the current collection mode. In addition, cloud service platform must strictly follow the user identity authentication procedures, it must be ensured that the system could only be accessed by legitimate users, which can be generally controlled by IP address, user account or login password etc.

\section{Set up security backup strategy for file data and make emergency plans}

Safety assessment team should be set up to manage the file data in colleges, they should master the security situation of archives information under the environment of "cloud storage", formulating scientific and reasonable evaluation index, to evaluate its security risk on a regular basis according to the evaluation index, and the contingency plans against the worst case shall be prepared in advance, while the accident disaster of the system, the loss could be reduced to the minimum. The files should be backup in advance for the archives with high importance and confidentiality, when the cloud database subject to fatal attack and damage, the backup system can be quickly started.

\section{The "private clouds" is recommended with better security status}

According to the distribution way and the range of service object, "cloud storage" can be divided into 3 categories, e.g. "public clouds", "private clouds" and "hybrid cloud". "Public clouds" is usually for large-scale and multi-user, but "private clouds" is running on a dedicated infrastructure of the data center, usually specific to a single user. In archives management of colleges, we prefer to choose "private cloud" service, at the same time of using cloud resources, the control right of archives could be maintained to the maximum extent.

4. The archives working style and service mode should be changed as soon as possible, the service quality should be further improved

"Cloud Archives" is a kind of technical practice of college archives management, but also a kind of brand-new management philosophy. Although this approach has professional technical personnel 
in the maintenance of "cloud storage" system for software and hardware equipments, the demand level of computer equipment and knowledge for archives management staff in colleges and universities is not high, but these staff should also learn and master the related knowledge and technology of "cloud storage" timely, renew the idea of the modernization of file storage, gradually adapt to the high-tech and modernization of archives management mode, so that the safety of the archives management could be realized, reliable, efficient and fast. The colleges can carry out various training courses and workshops of "cloud storage" to improve the comprehensive quality of archives management, thus further improving the quality of service.

\section{Conclusion}

The rapid development of "Cloud storage" technology provides a new platform and development opportunities for the archives information storage, backup and utilization, pointing out the new development direction for the archives work of college. The masses of the archivists in colleges should take advantage of the cloud to develop larger and more efficient file sharing space, based on their own archives resources and hardware facilities, combined with their own soft environment of archives development, continuously fulfilling the demand of archives information resources from the colleges the social, fully play the functions of archives information resources to the maximum level, promote the sustainable development of the college archives business, and make greater contribution to the management level of colleges to a new step.

\section{References}

[1] Shen ZhenFeng. The risks of archive information management system and safety protection in cloud era [J]. Journal of Taiwan world, 2013 (z6).

[2] Li mei. A study for new approach of using cloud storage as electronic archives [J]. Journal of Science \& Technology - electronic (middle), 2013 (10).

[3] Zhao Yi. Opportunities and risks: Electronic document management under cloud computing environment [J]. Journal of Archives and construction, 2013 (10).

[4] Li Zhangshu. Analyses the application of university archives "cloud" [J]. Journal of Taiwan world, 2012 (35).

[5] wang. Archives application mode in cloud era [J]. Journal of wit, 2014 (14). 